INPLASY

PROTOCOL

To cite: Zeng et al. Evaluation of Integrated Neuromuscular Training on the Recovery of Joint Injury: A protocol for systematic review and meta analysis. Inplasy protocol

2021120136. doi:

10.37766/inplasy2021.12.0136

Received: 31 December 2021

Published: 31 December 2021

Corresponding author:

Qing Liu

1208676945@qq.com

Author Affiliation:

Chengdu Sport University.

Support: 21XJC890001.

Review Stage at time of this submission: Risk of bias assessment - Completed but not published.

\section{Evaluation of Integrated Neuromuscular Training on the Recovery of Joint Injury: A protocol for systematic review and meta analysis}

\author{
Zeng, J1; Liu, Q2; Lei, Z3; Sun, Z4; Wang, Y5.
}

Review question / Objective: This study will provide new evidence for the effect of integrated neuromuscular training on the recovery of joint injury.

Information sources: According to the PICOS principle, the third and fourth authors of this paper searched PsycINFO, Science direct, PubMed, Eric, Willey, China Knowledge Network (CNKI) Academic Journal Online Publishing General Library and China Knowledge Network (CNKI) excellent doctoral thesis full-text database by computer to collect relevant research on the impact of INT on joint injury repair. The time limit of injury retrieval is from the establishment of the database to December 2021.

INPLASY registration number: This protocol was registered with the International Platform of Registered Systematic Review and Meta-Analysis Protocols (INPLASY) on 31 December 2021 and was last updated on 05 January 2021 (registration number INPLASY2021120136).

\section{INTRODUCTION}

Review question / Objective: This study will provide new evidence for the effect of integrated neuromuscular training on the recovery of joint injury.

Condition being studied: Integrated neuromuscular training (INT) is a comprehensive and holistic training method. It combines general functional movement training with specialized strength, balance, speed, sensitivity, coordination, enhanced training or rapid telescopic compound training. The human body can effectively prevent human joint injury and promote the recovery effect after injury through integrated neuromuscular 
training effect. From the existing research results, the action mechanism of integrated neuromuscular training is mainly through the impact of integrated neuromuscular training on the motor sensory system, through the integration of multi sensory information to the domination of the central nervous system, and finally through the motor output of skeletal muscle, so as to realize local sensation, neuromuscular control, posture control Improve the ability of sports performance and sports injury prevention, so as to achieve the impact on the motor sensory system. Based on this, it is becoming more and more important to carry out integrated neuromuscular training to prevent joint injury and recover after joint injury. In the existing research results, neuromuscular training includes super isometric movement training, core strength and balance training, resistance strength training, intermittent speed training and sensitivity training. However, there are still some disputes about the training effect and training mechanism of int. There are still many differences between the conclusions of the research on the influence of int training on sprint ability, sensitivity and balance ability. The existing meta-analysis only focuses on the treatment and recovery of ACL injury by INT, the research on other joints of lower limbs is relatively weak, and there is almost no relevant research on upper limbs and spinal column joints. In addition, the impact of INT cycle, intervention frequency and intervention time on joint injury, the differences of intervention effects among different populations, ages and genders, and whether different test methods and methods can accurately evaluate and reflect the recovery of injured populations are also worth studying. Based on this, this study uses the meta analysis method to systematically analyze the RCT tests related to the influence of INT on joint injury at home and abroad, and test the recovery effect of INT on joint injury, in order to provide reference for the practice of the recovery effect of INT on different joint injuries.Integrated neuromuscular training (INT) is a comprehensive and holistic training method. It combines general functional movement training with specialized strength, balance, speed, sensitivity, coordination, enhanced training or rapid telescopic compound training. From the existing research results, the mechanism of integrated neuromuscular training mainly lies in improving the proprioception of the human body And cognitive level to achieve the impact on the motor sensory system, so as to effectively prevent joint injury and promote the recovery after joint injury.

\section{METHODS}

Search strategy: The references of the retrieved literature are traced to supplement the relevant literature. The retrieval adopts the combination of subject words and free words. The Keywords are "integrated neuromuscular training", "joint injury" and "recovery" et al; Taking CNKI search library as an example. The specific search strategy of this study is shown in Box 1. The same strategies are used in other electronic databases. Box1 CNKI searching \#1 Integrated neuromuscular training \#2 Joint injury \#3 Knee joint injury \#4 Hip joint injury \#5 Ankle joint injury \#6 Shoulder joint injury \#7 Elbow joint injury \#8 Wrist joints injury \#9 Thoracic vertebra injury \#10 Lumbar vertebra injury \#11 Cervical vertebra injury.

Participant or population: Patients with joint injury.

Intervention: The experimental group received medical treatments and INT intervention scheme after medical treatments. The control group only received the medical treatments. We will study the effect of integrated neuromuscular training on joint injury. The study will be selected according to the following criteria:(1) study type: Randomized controlled trial.

Comparator: Test the range of motion of the patient's joints.

Study designs to be included: We will study the effect of integrated neuromuscular training on joint injury. The study will be selected according to the following criteria: 
(1) study type: Randomized controlled trial. The experimental group received medical treatments and INT intervention scheme after medical treatments. The control group only received the medical treatments.

Eligibility criteria: Review and comment research or non Chinese and English literature; In the study, only the experimental group, no control group or the control group is the literature of blank control; Literature published in the form of abstracts, research that cannot obtain the full text, or literature with incomplete research data and unsuccessful contact with the author.

Information sources: According to the PICOS principle, the third and fourth authors of this paper searched PsycINFO, Science direct, PubMed, Eric, Willey, China Knowledge Network (CNKI) Academic Journal Online Publishing General Library and China Knowledge Network (CNKI) excellent doctoral thesis full-text database by computer to collect relevant research on the impact of INT on joint injury repair. The time limit of injury retrieval is from the establishment of the database to December 2021.

Main outcome(s): Due to joint injury and recovery is a global problem, we should pay attention to non English language analysis in order to ensure the accuracy of the conclusion, so that other scholars can further study recovery effect of sports medicine on joint injury.

Quality assessment / Risk of bias analysis: Publication bias means that the research results with statistical significance are easier to publish, while the research results without statistical significance are often rejected, which makes it difficult to collect the literature without statistical significance in the meta-analysis process, resulting in systematic errors between the included research and the actual research, and then affect the meta-analysis results. In order to ensure the quality and effectiveness of the included literature, the bias risk of the included literature was comprehensively evaluated according to the evaluation tool for the bias risk of randomized controlled trials in the Cochrane system evaluator manual. The study used Review Manager 5.3 software to evaluate the methodological quality of the sources of bias (Selective bias, implementation bias, measurement bias, loss of follow-up bias and other bias) included in the literature.

Strategy of data synthesis: The horizontal axis of funnel chart is the standardized mean difference (Effect quantity), and the vertical axis is its standard error. If the effect quantity of funnel diagram is evenly and symmetrically distributed on the left and right, it indicates that meta-analysis has no publication bias, on the contrary, it has publication bias. Rosenthal's loss of safety factor (Nfs), egger linear regression test and shear compensation method were further used to test publication bias. First, if NFS is less than the critical value $5 K+10$ ( $K$ refers to the number of independent effects included in the meta-analysis), it indicates that there may be publication deviation. (Table 2)

Subgroup analysis: The random effect model was used for subgroup analysis to investigate the role of three regulatory variables: treatment mode, treatment time and disease cycle in the recovery effect of integrated neuromuscular training on joint injury.

Sensitivity analysis: Sensitivity analysis was used to analyze the research quality, intervention methods, publication types, etc. If the heterogeneity is large, the method of eliminating the literature one by one should be used for sensitivity analysis.

\section{Country(ies) involved: China.}

Keywords: Integrated neuromuscular training; Joint injury; Restoration; Meta analysis; System evaluation.

Conflicts of interest: This systematic review is funded by the Youth Foundation for Humanities and Social Sciences Research of the Ministry of Education(Funding 
reference number is 21 XJC890001)

supported by the foundation.

Contributions of each author:

Author 1 - Jing Zeng.

Email: 1208676945@qq.com

Author 2 - Qing Liu.

Author 3 - Zhengfang Lei.

Author 4 - Zhe Sun.

Author 5 - Yang Wang. 